\title{
Calculation of frontier orbitals, electronic coupling and paramagnetic interaction of conjugated porphyrin arrays based on DFT theory
}

Francesca Ginia a, Federica Foliab ${ }^{\text {a }}$, Aria M. Maruccoc ${ }^{\text {b }}$, Jason Facchetti a, Ivan Fenoglioc $^{a^{*}}$, Loreal Serpeb ${ }^{b}$

${ }^{\text {a } U n i v e r s i d a d e ~ F e d e r a l ~ d e ~ S a ̃ o ~ P a u l o, ~ L a b o r a t o ́ r i o ~ d e ~ L a s e r s ~ e ~ O ́ p t i c a ~ B i o m e ́ d i c a ~ A p l i c a d a, ~ D e p a r t a m e n t o ~}$ de Física, Diadema, SP, Brazil

${ }^{\mathrm{b}}$ Centro de Lasers e Aplicações, IPEN-CNEN/SP, São Paulo, SP, Brazil

\begin{abstract}
Electronic donor-acceptor communication in porphyrin-based molecular electronics is addressed theoretically. The order of the $\mathrm{a}_{1 \mathrm{u}}$ and $\mathrm{a}_{2 \mathrm{u}}$ frontier occupied orbitals has been proposed by others to affect excited energy transfer. We examined the effects of the nature and pattern of substituents upon analog monomer porphyrin orbitals using DFT (B3LYP/6-31G(d,p)) and the four-orbital model of porphyrin electronic structure. The excited state energy transfer rate was found to be very sensitive to these orbitals' contributions to porphyrin uv/visible absorptions (B- and Q-band). Ground state homodimer calculations validated the monomer results.
\end{abstract}

\section{Introduction}

Molecular electronics has garnered much attention ${ }^{1-2}$. Understanding the electronic communication between a donor and acceptor pair is critical for the rational design of nanoscale molecular electronic devices. Recently, several prototypical molecular electronic elements were made using porphyrins as building blocks. ${ }^{3-15}$ One key fact is that the ordering of the porphyrin frontier orbitals plays a crucial role in determining the rates of electronic communication. ${ }^{2,16-20,15}$ Moreover, the variation in electron transfer rates was proposed to arise from different ordering of the HOMO and HOMO1 orbitals For example, high density would be associated with efficient interporphyrin electronic communication, and thus rapid electron transfer. Similar results were found for ground state electron/hole hopping rates in fluorinated and unfluorinated zinc porphyrin homodimers. $^{21}$

The two highest occupied metalloporphyrin orbitals, $\mathrm{a}_{1 \mathrm{u}}$ and $\mathrm{a}_{2 \mathrm{u}}$, are nearly degenerate, but have substantially different electronic distributions (Fig. 2). The $\mathrm{a}_{2 \mathrm{u}}$ orbital is heavily populated at the meso-carbons but has only slight density at the $\beta$-carbons. In contrast, the $\mathrm{a}_{1 \mathrm{u}}$ orbital has virtually no electron density at the meso-carbons but has a large population at the $\beta$-carbons. ET photoinduced in resonance with the Q-band 
region of the spectrum is dominated by the HOMO (see below). For meso-linked systems, then, having $\mathrm{a}_{2 \mathrm{u}}=$ HOMO should result in faster ET than having $\mathrm{a}_{1 \mathrm{u}}=$ HOMO. The converse should be true for $\beta$-linked porphyrins.

In this work, we report upon an investigation of electronic communication in porphyrin dimers employing high-level DFT calculations and the Gouterman four-orbital model. In particular, studies of a series of monomer analogs of the ZnFbU- $m$ dimers have allowed a detailed exploration of the effects of the nature and pattern of peripheral substituents decorating the porphyrin macrocycle upon the frontier orbital energies. The relationship between the photoinduced ET rates and the frontier orbital energetics was explored by analyzing the contributions of each of the frontier orbitals to the observed uv/visible absorption transitions. In addition, challenging ground state calculations of homodimer zinc porphyrin systems were performed. Our results account for the experimental findings, and show a correlation between the experimental ET rates and the frontier orbital ordering and magnitude of energetic splitting. More importantly, this study provides a theoretical guideline for the design of porphyrin-based molecular electronics.

\section{Methods}

All quantum mechanical calculations were full geometry optimizations performed using GAUSSIAN 98W on a PC platform, employing symmetry only where appropriate. The B3LYP hybrid functional and a moderate Gaussian-type basis set $(6-31 G(d, p))$ were used.

One component of this study is the determination of the magnitudes of the contributions of relevant electronic configurations to the uv/visible electronic excitations of the $\mathrm{Zn}$ porphyrin: the B-band ( 400-450 nm) and the Q-band ( 550-600 nm) (Fig. 3). These two porphyrin absorption bands involve $\pi-\pi *$ transitions among the frontier orbitals, primarily the $\mathrm{a}_{1 \mathrm{u}}$ and $\mathrm{a}_{2 \mathrm{u}} \mathrm{Occupied}$ and the $\mathrm{e}_{\mathrm{g}}$ unoccupied orbitals. The magnitudes of the contributions depend upon several factors, including the ordering of the frontier molecular orbitals, the energy differences among them, and the configuration interaction $(\mathrm{CI})$ coupling strength. We developed a quantitative model to determine these magnitudes based upon the Gouterman four-orbital picture for porphyrin electronic structures, the eigenvalues associated with the frontier orbitals determined by our DFT calculations, and the experimentally observed absorption spectra, where available. 
(a)

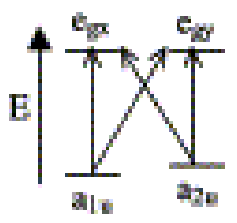

(b)

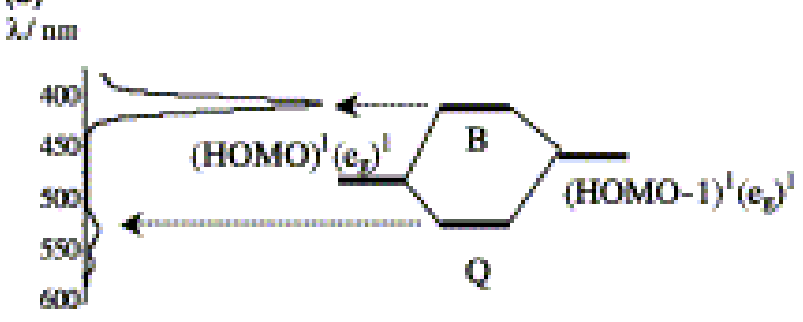

Fig. 3. Transitions and configuration interaction in the gouterman four orbital model. (a) Four allowed transitions, $\left(a_{1 u} \mathbf{e}_{g x}\right),\left(a_{2 u} \mathbf{e}_{g y}\right),\left(a_{1 u} \mathbf{e}_{g y}\right)$, and $\left(\mathbf{a}_{2 u} \mathbf{e}_{g y}\right)$, each with $E_{u} s y m m e t r y$, mix via configuration interaction. (b) The lower energy doubly degenerate $\mathrm{HOMO} \rightarrow \mathrm{e}_{\mathrm{g}}$ transition has a larger contribution to the Q-band, while the higher energy doubly degenerate (HOMO1) $\rightarrow e_{\mathrm{g}}$ transition has a larger contribution to the B-band. The absorption spectrum shown is $\mathrm{Zn}$ porphine in $\mathrm{CS}_{2}$.

The Gouterman four-orbital model assumes that only the $\left(\mathrm{a}_{2 \mathrm{u}}\right)^{1}\left(\mathrm{e}_{\mathrm{g}}\right)^{1}$ and $\left(\mathrm{a}_{\mathrm{u}}\right)^{1}\left(\mathrm{e}_{\mathrm{g}}\right)^{1}$ configurations participate in the Q- and B-bands (Fig. 3a). Both of these doubly degenerate excited state configurations have $E_{\mathrm{u}}$ symmetry, generating strong CI. Since these configurations are only nearly degenerate, their contributions to the Q- and B-bands are unequal. The lower energy configuration $(\mathrm{HOMO})^{1}\left(\mathrm{e}_{\mathrm{g}}\right)^{1}$ will contribute more to the Q-band, and the higher energy (HOMO-1) ${ }^{1}\left(\mathrm{e}_{\mathrm{g}}\right)^{1}$ more to the B-band (Fig. $3 b$ ).With similar expressions for $y$-polarized transitions [16]. $B_{x}{ }^{0}$ and $Q_{x}{ }^{0}$ are a basis of 50-50 mixtures of the $\left(\mathrm{a}_{1 \mathrm{u}}\right)^{1}\left(\mathrm{e}_{\mathrm{g}}\right)^{1}$ and $\left(\mathrm{a}_{2 \mathrm{u}}\right)^{1}\left(\mathrm{e}_{\mathrm{g}}\right)^{1}$ configurations. That is, if the $\mathrm{a}_{1 \mathrm{u}}$ and $\mathrm{a}_{2 \mathrm{u}}$ orbitals are (accidentally) exactly degenerate for the $\mathrm{D}_{4 \mathrm{~h}}$ porphyrin, then $B_{x}=B_{x}{ }^{0}$ and $Q_{x}=Q_{x}{ }^{0}$. The unmixing coefficient $\theta_{x}$ that relieves their non-degeneracy. For these initial studies, the two lowest energy LUMOs were assumed to be degenerate, as is strictly true, by symmetry, for $\mathrm{D}_{4 \mathrm{~h}}$ porphyrins. Under this assumption, the measured absorption spectrum and B3LYP/6-31G(d,p) energies for the top two HOMOs and lowest two LUMOs are used to calculate the CI coupling strength. The absorption spectrum of $\mathrm{F}_{10}-\mathrm{ZnTPP}$ is not known, therefore a value of $\mathrm{C}=3200 \mathrm{~cm}-1$ was used in the calculating the molecular orbital contributions to the excited state. This value is an average from calculations for species whose absorption spectra are known.

\section{Results and discussion}

The goal of these studies was to examine the dependence of photoinduced ET rates in porphyrin arrays upon the electronic structure of the system. For porphyrins, the absorption bands used to photoinduce ET derive primarily from strong CI mixing of four electronic excitations involving the occupied $\mathrm{a}_{1 \mathrm{u}}$ and $\mathrm{a}_{2 \mathrm{u}}$ orbitals and the doubly degenerate unoccupied egorbitals (Fig. 3). Therefore, these studies began by exploring the energies of these frontier orbitals in monomer analogs of the $\mathrm{ZnFbU}$ dimer (Fig. 1). 
We focused upon Q-band excitation of the meso-linked systems, though the approach applies equally well to the $\beta$-linked dimers and to excitation in the B-band.

The B3LYP hybrid density functional and a moderate, Gaussian-type basis $(6-31 \mathrm{G}(\mathrm{d}, \mathrm{p}))$ were used to perform full geometry optimizations on the set of porphyrins depicted in Fig. 1b. The DFT was employed for several reasons. By their nature, DFT methods address the electron density directly. In fact, unlike Hartree-Fock (HF) theory, the Kohn-Sham (KS) orbitals, in principle, can give the exact ground state electron density. In addition the KS orbitals are based upon a one-electron potential that includes correlation. Hence DFT methods are less expensive (time, memory) than HF-based methods at correlated levels. Finally, the KS orbitals have been recently found to be valuable, and perhaps even better than HF-based molecular orbitals, when used in the molecular orbital picture. Orbital ordering and energy differences are calculated well. In particular, the B3LYP functional, which was parameterized for accuracy in calculating energetic observables, has been widely successful.

Fig. 4, Fig. 5 show some results of B3LYP/6-31G(d,p) calculations on substituted zinc porphyrins. Zn porphine is the simplest zinc porphyrin, with hydrogens at all 12 peripheral sites on the macrocycle. The $\mathrm{a}_{1 \mathrm{u}}$ and $\mathrm{a}_{2 \mathrm{u}}$ orbitals were found to be nearly degenerate in $\mathrm{Zn}$ porphine, with $\mathrm{a}_{1 \mathrm{u}}$ the HOMO by only $0.12 \mathrm{kcal} / \mathrm{mol}$ (Fig. 4). Upon replacement of the four meso-hydrogens with electron-donating phenyl groups (ZnTPP (3)), the $\mathrm{a}_{2 u}$ orbital was preferentially destabilized, making it the HOMO by 1.7 $\mathrm{kcal} / \mathrm{mol}$. Increasing the electron-donating capacity of the phenyls by substitution of $\mathrm{NH}_{2}$ groups at the ortho positions $\left(\left(o, o^{\prime}-\mathrm{NH}_{2}\right)_{8}-\mathrm{ZnTPP}(4)\right)$ further destabilized the $\mathrm{a}_{2 \mathrm{u}}$ orbital.

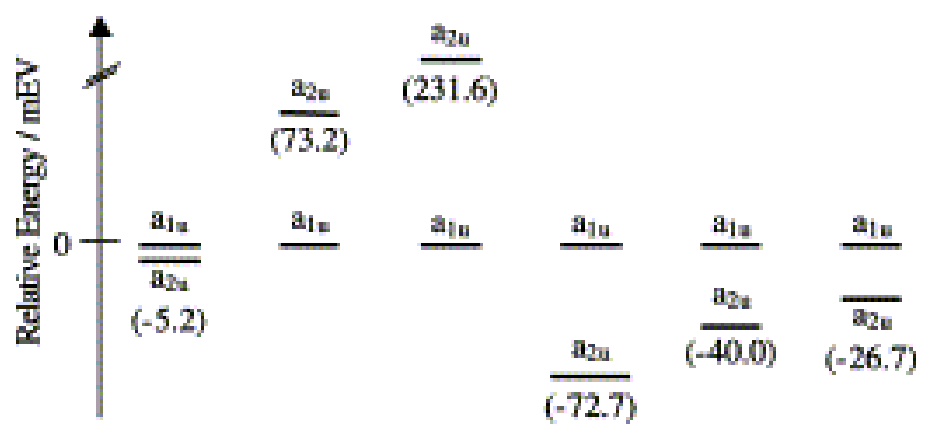
(a)
(b)
(c)
(d)
(e)
$(6)$

Fig. 4. Relative $a_{1 u}$ and $a_{2 u}$ energies for several substituted zinc porphyrins. (a) $\mathrm{Zn}$ porphine; (b) ZnTPP (3); (c) ZnTPP with $-\mathrm{NH}_{2}$ at phenyl ortho positions (4); (d) $\mathbf{F}_{20}-\mathrm{ZnTPP}$ (5a); (e) $\mathrm{Cl}_{20}$-ZnTPP (6); (f) $\mathrm{Br}_{20}-\mathrm{ZnTPP}$ (7). Full geometry optimizations were performed at the B3LYP/6-31G(d,p) level. The numbers in parentheses are the energies of the $a_{2 u}$ orbitals relative to the $\mathrm{a}_{1 \mathrm{u}}$. 


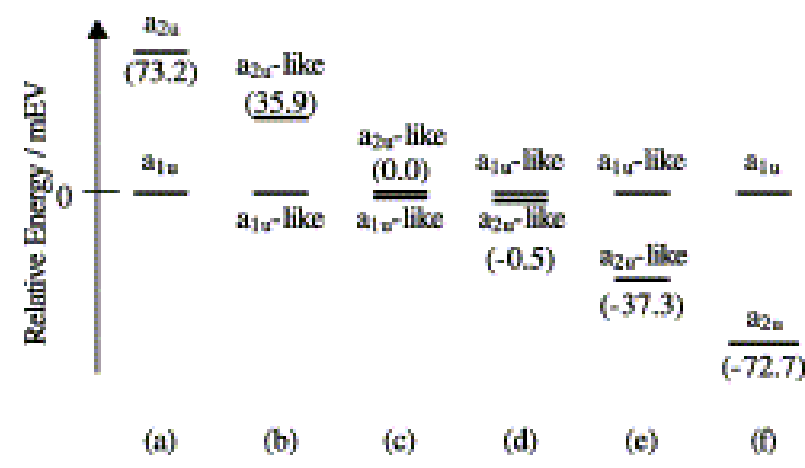

Fig. 5. Relative $a_{1 u}$ and $a_{2 u}$ energies for a series of fluorinated ZnTPPs. ZnTPP with 0 (a), 1 (b), 2 ((c) = across macrocycle, $(\mathrm{d})=$ adjacent on macrocycle), 3 (e) or 4 (f) pentafluorinated meso-phenyls (5a-5e). Full geometry optimizations were performed at the B3LYP/6-31G(d,p) level. The numbers in parentheses are the energies of the $\mathbf{a}_{2 u}$ orbitals relative to the $\mathbf{a}_{1 \mathrm{u}}$.

The use of electron-withdrawing groups had the predicted effect of preferentially stabilizing the $a_{2 u}$ orbital relative to the $a_{1}$. By tuning the degree of electron withdrawing power, the ordering and splitting of the frontier orbitals could be easily and nearly continuously altered. For example, by pentafluorinating the phenyl groups in $\mathrm{ZnTPP}$ to form $\mathrm{F}_{20}-\mathrm{ZnTPP}(\mathbf{5 a})$, the $\mathrm{a}_{2 \mathrm{u}}$ orbital, which was the HOMO in ZnTPP by $1.7 \mathrm{kcal} / \mathrm{mol}$, was stabilized to become the (HOMO-1) in $\mathrm{F}_{20}-\mathrm{ZnTPP}$. Using less electronegative halogens reduced the degree of stabilization correspondingly, bringing the $\mathrm{a}_{2 \mathrm{u}}$ orbital closer in energy to the $\mathrm{a}_{1 \mathrm{u}}$ HOMO (Fig. 4).

The splitting and ordering could be tuned by changing number of electron-withdrawing groups as well. For example, the pentafluorophenyl series in Fig. 5 shows a wide range of ordering and splitting from ZnTPP to $\mathrm{F}_{20}-\mathrm{ZnTPP}$. Each pentafluorophenyl group additively stabilized the $\mathrm{a}_{2 \mathrm{u}}$ relative to the $\mathrm{a}_{1 \mathrm{u}}$ orbital by approximately $0.85 \mathrm{kcal} / \mathrm{mol}$. ZnTPP and $\mathrm{F}_{5}-\mathrm{ZnTPP}$ (5e) had an a a orbital as the HOMO. With two pentafluorophenyl groups and two phenyl groups $\left(\mathrm{F}_{10}-\mathrm{ZnTPP}\right)$, the $\mathrm{a}_{1 \mathrm{u}}$ and $\mathrm{a}_{2 \mathrm{u}}$ orbitals were nearly degenerate, regardless of whether the pentafluorophenyls were across the macrocycle (5d) from each other or at adjacent meso-positions (5c). Finally, in $\mathrm{F}_{15}$ ZnTPP (5b) and $\mathrm{F}_{20}-\mathrm{ZnTPP}(\mathbf{5 a})$ the $\mathrm{a}_{2 \mathrm{u}}$ orbital experienced sufficient stabilization to drop below the $\mathrm{a}_{1 \mathrm{u}}$.

To understand the influence of the frontier orbitals upon ET, excited state wavefunctions must be explored. The ground state configuration of metalloporphyrins is either $\left(a_{1 u}\right)^{2}\left(a_{2 u}\right)^{2}\left(e_{g}\right)^{0}$ or $\left(a_{2 u}\right)^{2}\left(a_{1 u}\right)^{2}\left(e_{g}\right)^{0}$. The doubly degenerate $\left(a_{2 u}\right)^{1}\left(e_{g}\right)^{1}$ and $\left(\mathrm{a}_{1 \mathrm{u}}\right)^{1}\left(\mathrm{e}_{\mathrm{g}}\right)^{1}$ are the lowest energy $\pi-\pi *$ excitations (Fig. 3a). Both have $E_{\mathrm{u}}$ symmetry, yielding strong CI. The Gouterman four-orbital model assumes that only $\left(\mathrm{a}_{2 \mathrm{u}}\right)^{1}\left(\mathrm{e}_{\mathrm{g}}\right)^{1}$ and $\left(\mathrm{a}_{1 \mathrm{u}}\right)^{1}\left(\mathrm{e}_{\mathrm{g}}\right)^{1}$ interact to generate the observed Q- and B-bands. The lower energy configuration (HOMO) $)^{1}\left(\mathrm{e}_{\mathrm{g}}\right)^{1}$ will contribute more to the Q-band, and the (HOMO$1)^{1}\left(\mathrm{e}_{\mathrm{g}}\right)^{1}$ more to the B-band (Fig. 3b). These contributions, in turn, determine the electronic character at the linker carbon upon excitation. The HOMO therefore should dominate the Q-excited ET rates, and the HOMO-1 the B-excited rates.

The size of the contribution of the HOMO or HOMO-1 to the Q- and B-bands depends upon the ordering and energy gap between the $\mathrm{a}_{1 \mathrm{u}}$ and $\mathrm{a}_{2 \mathrm{u}}$ orbitals and the $\mathrm{CI}$ coupling 
strength. We have used our DFT results and experimental absorption spectra, where available, to quantify the relative contributions of each excited configuration $\left(\mathrm{a}_{1 \mathrm{u}}\right)^{1}\left(\mathrm{e}_{\mathrm{g}}\right)^{1}$ and $\left(\mathrm{a}_{2 \mathrm{u}}\right)^{1}\left(\mathrm{e}_{\mathrm{g}}\right)^{1}$ to the Q- and B-band excited states, within the framework of the Gouterman picture (Table 1).

For the meso-linked dimers, Q-excited ET rates should be slow when $\mathrm{a}_{1 \mathrm{u}}$ is the HOMO with a large component contributing to the Q-band, and fast when $\mathrm{a}_{2 \mathrm{u}}$ is HOMO with a large component. Our results support this hypothesis. For example, $\mathrm{F}_{30}-\mathrm{ZnFbU}-m$ has a relatively long ET lifetime of $240 \mathrm{ps}$. The analog of $\mathrm{F}_{30}-\mathrm{ZnFbU}-m$ is $\mathrm{F}_{15}-\mathrm{ZnTPP}$. This molecule was found to have an a $1 \mathrm{u}$ HOMO that contributes more heavily (50.9\%) to the Q-state. The ET rate is an order of magnitude faster for $\mathrm{ZnFbU}-m$, whose monomer analog ZnTPP has an $\mathrm{a}_{2 \mathrm{u}}$ HOMO that contributes $51.4 \%$ to the Q-state. Similarly positive correlations were observed for ET rates in the $\beta$-linked dimers.

One surprising finding is that the changes in contributions of the excited configurations to the Q- and B-states were relatively small. For example, the $\left(\mathrm{a}_{1 \mathrm{u}}\right)^{1}\left(\mathrm{e}_{\mathrm{g}}\right)^{1}$ contribution increased by only $2.3 \%$ from ZnTPP to $\mathrm{F}_{15}-\mathrm{ZnTPP}$. The large (order-of-magnitude) difference in ET rates for the analogous dimers suggests that the electronic communication is very sensitive to the details of the porphyrin excited states.

The quantification inherent in this approach allows us to correlate the configuration contribution with the ET rate, and to predict ET rates for unmeasured species. For example, $\mathrm{F}_{5}-\mathrm{ZnTPP}$ is the monomer analog for the $\mathrm{F}_{10}-\mathrm{ZnFbU}-m$ dimer. Assuming a simple linear relationship between the square of the configuration contribution and the ET lifetime, $\mathrm{F}_{10}-\mathrm{ZnFbU}-m$ should have a lifetime of 100 ps with Q-band excitation. Similarly, the predicted ET lifetime for $\mathrm{F}_{20}-\mathrm{ZnFbU}-m$ was determined to be $160 \mathrm{ps}$. Similar analyses can be applied to the $\beta$-linked systems. This simple linear relationship is not likely to be generally appropriate, as it predicts lifetimes of below zero for $\left(\mathrm{a}_{2 \mathrm{u}}\right)^{1}\left(\mathrm{e}_{\mathrm{g}}\right)^{1}$ components of $>51.7 \%$. However, since only two experimental points are available, it gives a reasonable starting point and predictions to which to compare future experiments and refine analyses.

The model also predicts that the ET rates should demonstrate wavelength dependence. First, there is a gross dependence upon the excited state in resonance with the exciting laser pulse. While the identity and contribution of the HOMO dominates the Q-band experiments, the rates measured from excitation in the B-band should be primarily influenced by the HOMO-1 orbital. A second type of wavelength dependence is more subtle, and arises from symmetry. Contrary to our assumption, the first two LUMOs in the porphyrins in dimers are not necessarily (i.e., by symmetry) degenerate. The $x$ and $y$-polarized transitions are therefore also not degenerate, and ET lifetimes should vary as the excitation source is tuned through the Q- or B-bands.

All of the calculations and correlations described thus far have relied upon high-level DFT calculations upon porphyrin monomers as analogs for dimer systems. We have also performed dimer calculations upon a limited subset of systems. Geometry optimizations under $\mathrm{D}_{2 \mathrm{~h}}$ symmetry constraints were carried out at the B3LYP/6$31 \mathrm{G}(\mathrm{d}, \mathrm{p})$ level for the ground states of the bis-ZnTPP dimers linked at the mesopositions $\left(\mathrm{Zn}_{2} \mathrm{U}-m\right.$ and $\left.\mathrm{F}_{30}-\mathrm{Zn}_{2} \mathrm{U}-m\right)$. The results show that the HOMO is a $\mathrm{a}_{2 \mathrm{u}}$-like for $\mathrm{Zn}_{2} \mathrm{U}-m$ and the splitting between the $\mathrm{a}_{2 \mathrm{u}}$-like and $\mathrm{a}_{1 \mathrm{u}}$-like orbitals is $1.6 \mathrm{kcal} / \mathrm{mol}$. As 
with the monomers, the HOMO of the perfluorinated complex was found to be $\mathrm{a}_{1 \mathrm{u}}$-like, with an $\mathrm{a}_{1 \mathrm{u}}-\mathrm{a}_{2 \mathrm{u}}$ energy splitting of $0.9 \mathrm{kcal} / \mathrm{mol}$. These results are very similar to those found for the ZnTPP $(1.7 \mathrm{kcal} / \mathrm{mol})$ and $\mathrm{F}_{15}-\mathrm{ZnTPP}(0.9 \mathrm{kcal} / \mathrm{mol})$ monomer analogs, respectively, and provide strong support for the soundness of our approach. Further dimer calculations are underway.

The orbital ordering and spacing of ZnTPP derivatives have been studied with high accuracy. We further quantify the relationship between frontier orbitals and the excited state configurations, and draw correlations to observed ET rates. The quantitative nature of the approach allows the model to be experimentally tested and refined. This approach may allow for the rational design of new porphyrin arrays with desired properties and photochemical responses.

\section{References}

1. Seidel, R.W. and I.M. Oppel, $1 D$ and 2D solid-state metallosupramolecular arrays of freebase 5,10,15,20-tetra(4-pyridyl)porphyrin, peripherally linked by zinc and manganese ions. Structural Chemistry, 2009. 20(1): p. 121-128.

2. Niu, W.Y., et al., 2D l-Di-toluoyl-tartaric acid Lanthanide Coordination Polymers: Toward Single-component White-Light and NIR Luminescent Materials. Chemistry-an Asian Journal, 2016. 11(4): p. 555-560.

3. Seidel, R.W. and I.M. Oppel, 2D Metallosupramolecular Arrays of Zinc-5,10,15,20-tetra(4pyridyl)porphyrin via Self-complementary Coordination and Peripheral Linkage. Zeitschrift Fur Anorganische Und Allgemeine Chemie, 2010. 636(3-4): p. 446-448.

4. Inoue, A., et al., 4-(dicyanomethylene)-2-methyl-6-(p-dimethylaminostyryl)-4H-pyran (DCM)doping density dependence of luminescence spectra and white emission in polymer lightemitting diodes. Physica Status Solidi C - Current Topics in Solid State Physics, Vol 6, No 1, 2009. 6(1): p. 334-337.

5. Han, C., et al., 5,10,15,20-tetrakis(4-chlorophenyl)porphyrin decorated TiO2 nanotube arrays: Composite photoelectrodes for visible photocurrent generation and simultaneous degradation of organic pollutant. Materials Science in Semiconductor Processing, 2016. 56: p. 166-173.

6. $\quad$ Elgazzar, S., et al., Ab initio computational and experimental investigation of the electronic structure of actinide 218 materials. Physical Review B, 2010. 81(23).

7. McDonald, J.F., et al., Accurate prediction of optimal cancer drug therapies from molecular profiles by a machine-learning algorithm. Journal of Clinical Oncology, 2015. 33(15).

8. Huang, J.S., et al., Achieving high-efficiency polymer white-light-emitting devices. Advanced Materials, 2006. 18(1): p. 114-117.

9. Botu, V. and R. Ramprasad, Adaptive machine learning framework to accelerate ab initio molecular dynamics. International Journal of Quantum Chemistry, 2015. 115(16): p. 1074-1083.

10. Li, A., et al., Aggregation Behavior of the Template-Removed 5,10,15,20-Tetrakis(4sulfonatophenyl)porphyrin Chiral Array Directed by Poly(ethylene glycol)-block-poly(L-lysine). Langmuir, 2014. 30(16): p. 4797-4805.

11. Wang, R.B., et al., Alkyne-Bridged Multi[Copper(II) Porphyrin] Structures: Nuances of Orbital Symmetry in Long-Range, Through-Bond Mediated, Isotropic Spin Exchange Interactions. 
Journal of the American Chemical Society, 2017. 139(29): p. 9759-9762.

12. Li, Z.Z., et al., All-organic flexible fabric antenna for wearable electronics. Journal of Materials Chemistry C, 2020. 8(17): p. 5662-5667.

13. Lee, J.S., et al., All-solution-processed high-brightness hybrid white quantum-dot light-emitting devices utilizing polymer modified quantum dots. Organic Electronics, 2017. 42: p. 393-398.

14. High, J.S., K.K. Virgil, and E. Jakubikova, Electronic Structure and Absorption Properties of Strongly Coupled Porphyrin-Perylene Arrays. Journal of Physical Chemistry A, 2015. 119(38): p. 9879-9888.

15. Freeman, A.J., Electronic-Structure Theory in the New-Age of Computational Materials Science. Annual Review of Materials Science, 1995. 25: p. 1-5.

16. Jiang, H. and W.P. Hu, The Emergence of Organic Single-Crystal Electronics. Angewandte Chemie-International Edition, 2020. 59(4): p. 1408-1428.

17. Carpenter, B.K., et al., Empirical Classification of Trajectory Data: An Opportunity for the Use of Machine Learning in Molecular Dynamics. Journal of Physical Chemistry B, 2018. 122(13): p. 3230-3241.

18. Zvezdin, A., et al., En route toward sustainable organic electronics. Mrs Energy \& Sustainability, 2020. 7.

19. Zhu, P.H., et al., An enhanced photoelectrochemical immunosensing platform: Supramolecular donor-acceptor arrays by assembly of porphyrin and C-60. Biosensors \& Bioelectronics, 2015. 68: p. 604-610.

20. Wang, R., et al., Enhanced white-light emission from multiple fluorophores encapsulated in a single layer of diblock copolymer micelles. Chemical Communications, 2011. 47: p. 2787-2789

21. Ma, Z., et al., Ensemble of machine learning algorithms using the stacked generalization approach to estimate the warfarin dose. PloS one, 2018. 13(10): p. e0205872.

22. Novikov, A.V., et al., Environment-friendly aqueous processing of [60]fullerene semiconducting films for truly green organic electronics. Journal of Materials Chemistry C, 2020. 8(2): p. 495499.

23. Li, J.L., et al., Evolution of Isoindigo-Based Electron-Deficient Units for Organic Electronics: From Natural Dyes to Organic Semiconductors. Asian Journal of Organic Chemistry, 2018. 7(11): p. 2147-2160.

24. Rawson, J., P.J. Angiolillo, and M.J. Therien, Exceptional delocalization of electrons in conjugated porphyrin arrays. Abstracts of Papers of the American Chemical Society, 2012. 244.

25. Yang, J., et al., Excitation energy migration processes in cyclic porphyrin Arrays probed by single molecule spectroscopy. Journal of the American Chemical Society, 2008. 130(6): p. 18791884.

26. Kim, P., et al., Excited-state energy relaxation dynamics of triply linked Zn(II) porphyrin arrays. Chemical Communications, 2011. 47(15): p. 4433-4435.

27. Kim, T., et al., Exciton coupling dynamics in syn- and anti-type beta-beta linked Zn(II) porphyrin linear arrays. Physical Chemistry Chemical Physics, 2016. 18(33): p. 23105-23110.

28. Hori, T., et al., Exploration of electronically interactive cyclic porphyrin arrays. Journal of Organometallic Chemistry, 2007. 692(1-3): p. 148-155.

29. Matsunaga, Y. and Y. Sugita, Linking time-series of single-molecule experiments with molecular dynamics simulations by machine learning. Elife, 2018. 7.

30. Kim, J.W., et al., Liquid coplanar-gate organic/graphene hybrid electronics for label-free 
detection of single and double-stranded DNA molecules. Organic Electronics, 2018. 62: p. 163167.

31. Vollbrecht, J., et al., Liquid crystalline dithienothiophene derivatives for organic electronics. Organic Electronics, 2018. 61: p. 266-275.

32. Tajima, K., Look beyond the surface: recent progress in applications of surface-segregated monolayers for organic electronics. Polymer Journal, 2019. 51(11): p. 1117-1126.

33. Klain, C., et al., Low work function Ca doped graphene as a transparent cathode for organic opto-electronics and OLEDs. Carbon, 2020. 157: p. 255-261.

34. Bullard, G., et al., Low-Resistance Molecular Wires Propagate Spin-Polarized Currents. Journal of the American Chemical Society, 2019. 141(37): p. 14707-14711.

35. Jamal, S., et al., Machine Learning and Molecular Dynamics Based Insights into Mode of Actions of Insulin Degrading Enzyme Modulators. Combinatorial Chemistry \& High Throughput Screening, 2017. 20(4): p. 279-291.

36. Karamzadeh, R., et al., Machine Learning and Network Analysis of Molecular Dynamics Trajectories Reveal Two Chains of Red/Ox-specific Residue Interactions in Human Protein Disulfide Isomerase. Scientific Reports, 2017. 7.

37. Majumder, A., et al., Machine Learning Approach for Argument Extraction of Bio-molecular Events. 2012 National Conference on Computing and Communication Systems (Ncces), 2012: p. 109-113.

38. Bolis, G., L. Dipace, and F. Fabrocini, A Machine Learning Approach to Computer-Aided Molecular Design. Journal of Computer-Aided Molecular Design, 1991. 5(6): p. 617-628.

39. Bartok, A.P., et al., Machine-learning approach for one- and two-body corrections to density functional theory: Applications to molecular and condensed water. Physical Review B, 2013. 88(5).

40. Li, Z., et al., Machine-Learning Energy Gaps of Porphyrins with Molecular Graph Representations. Journal of Physical Chemistry A, 2018. 122(18): p. 4571-4578.

41. Peng, X.B., et al., Macroscopic Films of Porphyrin Nanowell-Arrays via Solvent DiffusionInduced Self-Assembly. Journal of Physical Chemistry C, 2010. 114(43): p. 18449-18454.

42. Lee, J.H., et al., Manipulation of discrete porphyrin-fullerene nanopillar arrays regulated by the phase separated infiltration of polymer in ternary blended organic thin-films. Solar Energy Materials and Solar Cells, 2015. 140: p. 428-438.

43. Mitra, K.Y., et al., Manufacturing of All Inkjet-Printed Organic Photovoltaic Cell Arrays and Evaluating Their Suitability for Flexible Electronics. Micromachines, 2018. 9(12).

44. Huang, B. and O.A. von Lilienfeld, Many-body representations for machine learning models of molecular properties. Abstracts of Papers of the American Chemical Society, 2016. 252.

45. Teo, R.D., et al., Mapping hole hopping escape routes in proteins. Proceedings of the National Academy of Sciences of the United States of America, 2019. 116(32): p. 15811-15816.

46. Emandi, G., et al., Merging Triptycene, BODIPY and Porphyrin Chemistry: Synthesis and Properties of Mono- and Trisubstituted Triptycene Dye Arrays. European Journal of Organic Chemistry, 2017(45): p. 6680-6692.

47. Thamyongkit, P., et al., Meso-C-13-labeled porphyrins for studies of ground-state hole transfer in multiporphyrin arrays. Journal of Organic Chemistry, 2007. 72(14): p. 5207-5217.

48. Mori, H. and A. Osuka, meso-meso Directly-linked trimeric and pentameric electron-deficient porphyrin-hexaphyrin hybrid arrays. Journal of Porphyrins and Phthalocyanines, 2016. 20(1- 
4): p. 245-253.

49. Morisue, M., et al., Self-complementary double-stranded porphyrin arrays assembled from an alternating pyridyl-porphyrin sequence. Chemical Science, 2015. 6(11): p. 6199-6206.

50. Rauch, V., et al., Self-organized porphyrin arrays on surfaces: the case of hydrophilic side chains and polar surfaces. Journal of Porphyrins and Phthalocyanines, 2014. 18(1-2): p. 67-75.

51. Park, S., et al., Self-powered ultra-flexible electronics via nano-grating-patterned organic photovoltaics. Nature, 2018. 561(7724): p. 516-+.

52. Kang, W.M., et al., Self-templating synthesis of a fluorescent porphyrin doped poly(methyl methacrylate) nano-array and its HCl gas sensing properties. Analytical Methods, 2016. 8(35): p. 6489-6493.

53. Wang, R., Significantly Improving the Prediction of Molecular Atomization Energies by an Ensemble of Machine Learning Algorithms and Rescanning Input Space: A Stacked Generalization Approach. Journal of Physical Chemistry C, 2018. 122(16): p. 8868-8873.

54. Riniker, S. and G.A. Landrum, Similarity maps - a visualization strategy for molecular fingerprints and machine-learning methods. Journal of Cheminformatics, 2013. 5.

55. Yang, F., et al., A Simple, Small-Bandgap Porphyrin-Based Conjugated Polymer for Application in Organic Electronics. Macromolecular Rapid Communications, 2018. 39(21).

56. Wang, R., et al., Simultaneous blue, green, and red emission from diblock copolymer micellar films: a new approach to white-light emission. Chemical Communications, 2009(44): p. 6723 6725.

57. Kim, D. and A. Osuka, Single-Molecule Photophysical Properties of Various Directly Linked Porphyrin Arrays. Multiporphyrin Arrays: Fundamentals and Applications, 2012: p. 1-54.

58. $\quad$ Park, M., et al., Single-molecule spectroscopic investigation of energy migration processes in cyclic porphyrin arrays. Journal of the American Chemical Society, 2007. 129(12): p. 35393544.

59. Pinkerton, N.M., et al., Single-Step Assembly of Multimodal Imaging Nanocarriers: MRI and Long-Wavelength Fluorescence Imaging. Advanced Healthcare Materials, 2015. 4(9): p. 13761385.

60. Shetti, V.S., Y. Pareek, and M. Ravikanth, Sn(IV) porphyrin scaffold for multiporphyrin arrays. Coordination Chemistry Reviews, 2012. 256(23-24): p. 2816-2842.

61. Dvivedi, A., Y. Pareek, and M. Ravikanth, Sn-IV Porphyrin Scaffolds for Axially Bonded Multiporphyrin Arrays: Synthesis and Structure Elucidation by NMR Studies. Chemistry-a European Journal, 2014. 20(15): p. 4481-4490.

62. Mardis, K.L., et al., Solution-State Conformational Ensemble of a Hexameric Porphyrin Array Characterized Using Molecular Dynamics and X-ray Scattering. Journal of Physical Chemistry A, 2009. 113(11): p. 2516-2523.

63. Maeda, K., M. Nitani, and M. Uno, Thermocompression bonding of conductive polymers for electrical connections in organic electronics. Polymer Journal, 2020. 52(4): p. 405-412.

64. Bouamaied, I. and E. Stulz, Thieme Chemistry Journal Awardees - Where are They Now? Stabilisation of Porphyrins in Tetranucleotide-Bisporphyrin Arrays by Duplex Formation with Peptide Nucleic Acid. Synlett, 2009(18): p. 2913-2918.

65. Zhang, C.G., et al., Ti-Substituted Boranes as Hydrogen Storage Materials: A Computational Quest for Ideal Combination of Stable Electronic Structure and Optimal Hydrogen Uptake. Chemistry-a European Journal, 2009. 15(24): p. 5910-5919. 
66. Wang, R.B., et al., Topology, Distance, and Orbital Symmetry Effects on Electronic Spin-Spin Couplings in Rigid Molecular Systems: Implications for Long-Distance Spin-Spin Interactions. Journal of Physical Chemistry A, 2020. 124(37): p. 7411-7415.

67. Hathout, R.M. and A.A. Metwally, Towards better modelling of drug-loading in solid lipid nanoparticles: Molecular dynamics, docking experiments and Gaussian Processes machine learning. European Journal of Pharmaceutics and Biopharmaceutics, 2016. 108: p. 262-268.

68. Zhao, Z.X., A.N. Cammidge, and M.J. Cook, Towards black chromophores: mu-oxo linked phthalocyanine-porphyrin dyads and phthalocyanine-subphthalocyanine dyad and triad arrays. Chemical Communications, 2009(48): p. 7530-7532.

69. Osuka, A., Towards meso-meso-Linked Porphyrin Arrays and meso-Aryl Expanded Porphyrins. Chemical Record, 2015. 15(1): p. 143-159.

70. Ventura, B., et al., Unusual Photoinduced Electron Transfer from a Zinc Porphyrin to a Tetrapyridyl Free-Base Porphyrin in a Noncovalent Multiporphyrin Array. Chemistry-a European Journal, 2010. 16(29): p. 8748-8756.

71. Bagchi, A., Use of Machine Learning Features to Detect Protein-Protein Interaction Sites at the Molecular Level. Information Systems Design and Intelligent Applications, Vol 2, 2015. 340: p. 49-54.

72. Stone, T.J., et al., The use of machine learning to develop a robust predictor of glioneuronal tumour molecular subtype. Neuropathology and Applied Neurobiology, 2018. 44: p. 35-35.

73. Abdel-Aal, R.E. and M. Raashid, Using abductive machine learning for online vibration monitoring of turbo molecular pumps. Shock and Vibration, 1999. 6(5-6): p. 253-265.

74. Leonardi, M.J., P.K.B. Palomaki, and P.H. Dinolfo, Using copper(I)-catalyzed azide-alkyne cycloaddition in the creation of triazole linked multi-porphyrin arrays. Abstracts of Papers of the American Chemical Society, 2010. 240.

75. Apostolakis, J., D. Hofmann, and T. Lengauer, Using simple learning machines to derive a new potential for molecular modeling. Rational Approaches to Drug Design, 2001: p. 125-134.

76. $\quad$ Bruce, R.C., et al., Valence Band Dependent Charge Transport in Bulk Molecular Electronic Devices Incorporating Highly Conjugated Multi-[(Porphinato)Metal] Oligomers. Journal of the American Chemical Society, 2016. 138(7): p. 2078-2081.

77. Korolkov, V.V., et al., van der Waals-Induced Chromatic Shifts in Hydrogen-Bonded TwoDimensional Porphyrin Arrays on Boron Nitride. Acs Nano, 2015. 9(10): p. 10347-10355.

78. Kim, K.S., et al., Various strategies for highly-efficient two-photon absorption in porphyrin arrays. Journal of Photochemistry and Photobiology C-Photochemistry Reviews, 2008. 9(1): p. 13-28.

79. Seo, D.G., et al., Versatile neuromorphic electronics by modulating synaptic decay of single organic synaptic transistor: From artificial neural networks to neuro-prosthetics. Nano Energy, 2019. 65 .

80. Zhang, L., et al., Virtual screening approach to identifying influenza virus neuraminidase inhibitors using molecular docking combined with machine-learning-based scoring function. Oncotarget, 2017. 8(47): p. 83142-83154.

81. Zhao, P. and L.L. Zhu, Virtual special issue: Organic and polymer materials for electronics. Chinese Chemical Letters, 2018. 29(12): p. 1706-1708. 